\title{
c-Flip overexpression affects satellite cell proliferation and promotes skeletal muscle aging
}

\author{
C Giampietri ${ }^{1,7}$, S Petrungaro ${ }^{1,7}$, P Coluccia ${ }^{2}$, F Antonangeli ${ }^{1}$, K Giannakakis ${ }^{3}$, T Faraggiana ${ }^{3,4}$, A Filippini $^{1}$, G Cossu ${ }^{5,6}$ and E Ziparo $^{\star, 1}$
}

This study shows that forcing c-Flip overexpression in undifferentiated skeletal myogenic cells in vivo results in early aging muscle phenotype. In the transgenic mice, adult muscle histology, histochemistry and biochemistry show strong alterations: reduction of fibers size and muscle mass, mitochondrial abnormalities, increase in protein oxidation and apoptosis markers and reduced AKT/GSK3 $\beta$ phosphorylation. In the infant, higher levels of Pax-7, PCNA, P-ERK and active-caspase-3 were observed, indicating enhanced proliferation and concomitant apoptosis of myogenic precursors. Increased proliferation correlated with NF- $\mathrm{kB}$ activation, detected as p65 phosphorylation, and with high levels of embryonic myosin heavy chain. Reduced regenerative potential after muscle damage in the adult and impaired fiber growth associated with reduced NFATc2 activation in the infant were also observed, indicating that the satellite cell pool is prematurely compromised. Altogether, these data show a role for c-Flip in modulating skeletal muscle phenotype by affecting the proliferative potential of undifferentiated cells. This finding indicates a novel additional mechanism through which c-Flip might possibly control tissue remodeling.

Cell Death and Disease (2010) 1, e38; doi:10.1038/cddis.2010.17; published online 29 April 2010

Subject Category: Internal Medicine

This is an open-access article distributed under the terms of the Creative Commons Attribution License, which permits distribution and reproduction in any medium, provided the original author and source are credited. This license does not permit commercial exploitation without specific permission.

Skeletal muscle is a complex tissue consisting predominantly of a single differentiated cell type, that is, the contractile, multinucleated myofiber. Quiescent cells, named satellite cells, are located underneath the basal lamina surrounding each myofiber. In the early postnatal life, satellite cells proliferate extensively and fuse to the fibers, contributing to muscle growth. Under physiological conditions, satellite cells are mitotically quiescent, whereas upon injury they proliferate and generate a pool of myoblasts that subsequently differentiate and fuse to repair damaged myofibers. During aging, skeletal muscle homeostasis is impaired and loss of both muscle bulk and regenerative ability occurs. Whether muscle regeneration in aged mice is modified by changes in the extrinsic environment or by alterations of the satellite cells themselves is still a controversial issue. ${ }^{1}$ However, it is generally accepted that skeletal muscle aging impairs satellite cell responses to activating stimuli and increases their sensitivity to apoptosis, leading to a significant decline in muscle mass and force. ${ }^{2}$ Features characterizing aged skeletal muscles include muscle atrophy, that is, decrease in both muscle mass and fiber cross-sectional area (CSA), shift to a slower phenotype, accumulation of oxidative-stress-induced somatic mutations in mitochondrial DNA (mtDNA), ${ }^{3}$ increase in apoptotic nuclei associated with increased caspase activation levels ${ }^{4}$ and inhibition of PI3K/Akt activation. ${ }^{5}$ Although skeletal muscle atrophy and other related changes are constantly present in aging, mechanisms affecting the regenerative potential of satellite cells are only partially clarified. ${ }^{6}$ Under normal conditions, satellite cell population maintains a relevant proliferative potential and is able to regenerate skeletal muscle throughout the organism lifespan, although a replicative senescence in aged skeletal muscle cannot be excluded. Under pathological conditions, such as in dystrophic muscles, satellite cell pool exhaustion may derive at least in part from the excessive proliferation during repeated cycles of degeneration and regeneration. ${ }^{7}$ Therefore, forced proliferation of satellite cells might be detrimental to regenerative potential. In this report, a link between forced proliferation and premature skeletal muscle aging has been elucidated by investigating a transgenic mouse model overexpressing in satellite cell

\footnotetext{
${ }^{1}$ Istituto Pasteur-Fondazione Cenci Bolognetti, Department of Histology and Medical Embryology, 'Sapienza' University of Rome, 00161 Rome, Italy; ${ }^{2}$ Department of Surgery 'P. Valdoni', 'Sapienza' University of Rome, 00161 Rome, Italy; ${ }^{3}$ Department of Experimental Medicine, 'Sapienza' University of Rome, 00161 Rome, Italy; ${ }^{4}$ Department of Histopathology, Instituto Dermopatico dell' Immacolata-IRCCS, 00167 Rome, Italy; ${ }^{5}$ Division of Regenerative Medicine, San Raffaele Scientific Institute, 20132 Milan, Italy and ${ }^{6}$ Department of Biology, University of Milan, 20129 Milan, Italy

${ }^{*}$ Corresponding author: E Ziparo, Department of Histology and Medical Embryology, 'Sapienza' University of Rome, Via A. Scarpa, 1400161 Rome, Italy.

Tel: + 390649 766586; Fax: + 390644 62854; E-mail: elio.ziparo@uniroma1.it

${ }^{7}$ These authors contributed equally to this work.

Keywords: transgenic mouse; myogenesis; atrophy; apoptosis

Abbreviations: CSA, cross-sectional area; d.a.b., days after birth; eMHC, embryonic myosin heavy chain; GAPDH, glyceraldehyde-3-phosphate dehydrogenase; P-, phosphorylated; SDH, succinate dehydrohenase; TA, tibialis anterior; Tg, transgenic; WT, wild type; mtDNA, mitochondrial DNA; EGFP, enhanced green fluorescent protein; SDH, succinate dehydrogenase; PCNA, proliferating cell nuclear antigen

Received 17.3.10; accepted 26.3.10; Edited by M Piacentini
} 
c-Flip protein, a well-known apoptosis regulator and proliferation inducer. c-Flip is a key molecule involved in Fas/Fas ligand system-dependent apoptosis and exists as a long splicing isoform (c-Flip $\mathrm{L}_{\mathrm{L}}$ ), short splicing isoforms (c-Flip $_{S}$ and $c-$ Flip $_{R}$ ) and cleavage variants ${ }^{8}$ deriving from caspase-dependent $\mathrm{c}$-Flip $\mathrm{L}_{\mathrm{L}}$ proteolysis. c-Flip proteins usually suppress apoptosis by inhibiting caspase- 8 activation through a heterodimerization process. ${ }^{9-11}$ Although the antiapoptotic function of $\mathrm{C}-\mathrm{Flip}_{\mathrm{L}}$ has been established, the proapoptotic activity of $\mathrm{C}-\mathrm{Flip}_{\mathrm{L}}$ overexpression has also been described. ${ }^{12} \mathrm{c}-\mathrm{Flip}_{\mathrm{L}}$ cleavage products mediate growth signals in different cell types through NF- $\mathrm{KB}$ and ERK. ${ }^{13}$ We have recently shown that $\mathrm{c}-\mathrm{Flip}_{\mathrm{L}}$ interferes with Akt/GSK3 $\beta$ signaling, inhibiting cardiac hypertrophy in response to pressure overload. ${ }^{14}$ In this study, by further investigating the transgenic $\mathrm{c}-\mathrm{Flip}_{\mathrm{L}}$ mouse model, ${ }^{14}$ we extend to skeletal muscle the effects of c-Flip on Akt/GSK3 $\beta$ signaling and show increased phosphorylation of NFATc2, a crucial factor regulating muscle cell size. ${ }^{15}$ c-Flip overexpression was found to lead to increased proliferation of satellite cells in the infant skeletal muscles and to several alterations of adult skeletal muscles evidenced at morphological, histochemical and molecular levels. Interestingly, such modifications are similar to the ones described in age-advanced muscles, leading us to conclude that $\mathrm{C}-\mathrm{Flip}_{\mathrm{L}}$ overexpression in undifferentiated muscle cells interferes with skeletal muscle homeostasis, inducing phenotypic signs of skeletal muscle premature aging.

\section{Results}

Promoter activation in skeletal muscle of c-Flip transgenic mouse. We have previously described a transgenic (Tg) mouse model expressing both mouse $c-$ Flip $_{\mathrm{L}}$ and enhanced green fluorescent protein (EGFP) in the cardiac muscle under transcriptional control of the $400 \mathrm{bp}$ trStra8 promoter in a unique bicistronic transcript. ${ }^{14}$ In the same model we then investigated, in two independent lines, the transgene expression in skeletal muscle through EGFP RT-PCR. EGFP was adopted as transgene expression marker as the endogenous and transgene c-Flip were difficult to discriminate. In fact, the expression level of c-Flip was not strongly increased in the adult Tg whole muscle when compared with the wild type (WT) at western blot level (Supplementary Figure 1). EGFP cDNA was present in both infant and adult Tg quadriceps, although at a higher level in the infant (Figure 1A). We explored the possibility that it reflected the expression of the transgene by a cell subpopulation. Western blot analyses showed that expression of low-molecular-weight $\mathrm{C}-\mathrm{Flip}_{\mathrm{L}}$ cleaved forms in infant $\mathrm{Tg}$ skeletal muscle was strongly increased when compared with WT, as detected by a monoclonal anti-c-Flip antibody that is able to recognize all cleaved forms of $\mathrm{C}-\mathrm{Flip}_{\mathrm{L}}$ (Figure 1B). To address the transgene cell expression pattern, EGFP immunohistochemical localization on both infant and adult quadriceps was then investigated. In the infant, $20 \%$ of myofiber nuclei were found to express EGFP, whereas in the adult the cells expressing EGFP were found outside the dystrophin ring and myofibers were negative
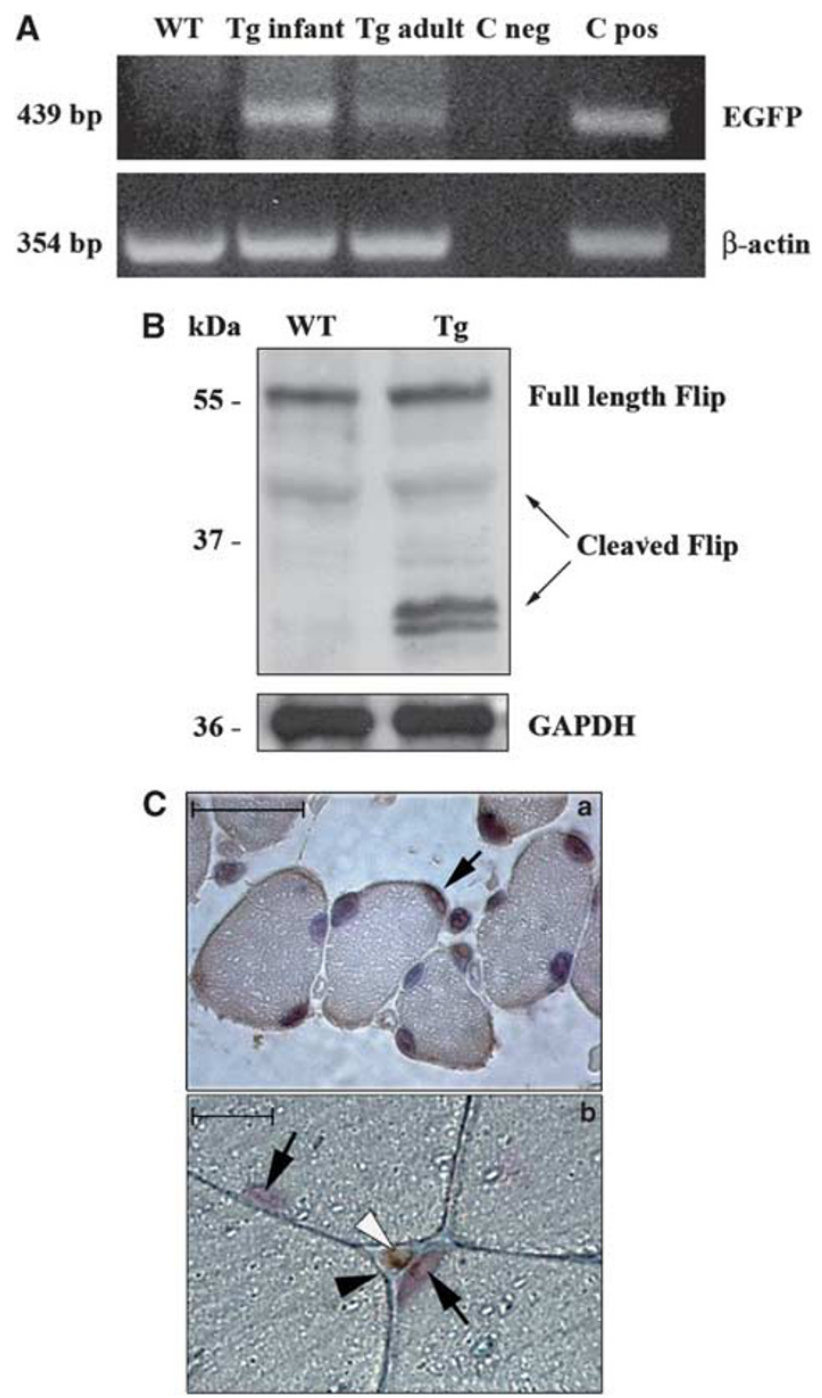

Figure 1 (A) Transgene expression. (Upper panel) Expression of EGFP mRNA is shown in both $\mathrm{Tg}$ infant quadriceps and $\mathrm{Tg}$ adult quadriceps, whereas it is undetectable in the WT quadriceps. $C$ neg represents negative PCR control (lacking C-DNA template). C pos corresponds to the positive control (adult heart). (Lower panel) Expression of $\beta$-actin mRNA in samples and controls. Data shown are representative of four independent experiments. (B, upper panel). C-Flip protein level increase in infant Tg quadriceps when compared with the WT. (Lower panel) GAPDH staining is shown as loading control. Data shown are representative of four independent experiments. (C) Immunohistochemistry anti-EGFP on Tg infant quadriceps sections. Brown cells (arrow) represent EGFP-positive cells. Sections were counterstained with hemalum. Bar: $50 \mu \mathrm{m}$ (a). Immunohistochemical labeling with anti-EGFP and anti-dystrophin antibodies on Tg adult quadriceps sections. The white arrowhead indicates one EGFP-positive cell, the black arrowhead indicates the dystrophin ring whereas the black arrows indicate hemalum counterstained myonuclei. Bar: $10 \mu \mathrm{m}$ (b)

for EGFP (Figure 1C). To further verify promoter activation in myogenic precursors, trStra8-EGFP vectors were transfected in the proliferating satellite cell-derived murine cell line $\mathrm{C} 2 \mathrm{C} 12$, and EGFP expression confirmed promoter activity in such cell type (Supplementary Figure 2). The transgene is therefore expressed primarily in satellite cells but not in mature myofibers. 
Histological, histochemical and biochemical analysis of adult transgenic skeletal muscle. Histological analyses of quadriceps skeletal muscles from $\mathrm{Tg}$ and WT mice were performed. At 4 months of age, WT and Tg muscles seem structurally similar (Figure 2A). In fact, no signs of inflammation or necrosis, or increased interstitial connective tissue are evident in $\mathrm{Tg}$ muscle. Nevertheless, frequency histograms of single-fiber CSA showed different size distribution in muscles from Tg versus WT mice with a shift toward smaller areas in the Tg (Figure 2B). In particular, the muscles from $\mathrm{Tg}$ mice showed an average $47.3 \%$ fiber size reduction, together with significant reduction of muscle mass when compared with the WT $(138 \pm 25$ versus $172 \pm 9 \mathrm{mg})$. Histochemical and biochemical studies were then performed to better characterize the phenotype of transgenic skeletal muscle. Alterations of mitochondrial electron transport system enzymatic activities have been frequently described in atrophic and aged muscles, ${ }^{16}$ and hence we investigated the activity of succinate dehydrogenase (SDH), a nuclearencoded mitochondrial enzyme that commonly increases under such conditions. Remarkably higher SDH activity in $\mathrm{Tg}$ mouse when compared with the WT skeletal muscles fibers is shown in Figures $3 \mathrm{~A}$ and $\mathrm{B}$, indicating a metabolic shift to more aerobic-oxidative metabolism, which is a hallmark of aged skeletal muscle. ${ }^{3}$ In addition, ultrastructural analysis of skeletal muscle indicated signs of irregular myofibrillar organization, together with well apparent increased mitochondrial mass in the subsarcolemmal region and many enlarged mitochondria, as shown in Figure $3 \mathrm{C}$. The increase in the cytochrome $c$ protein expression is commonly
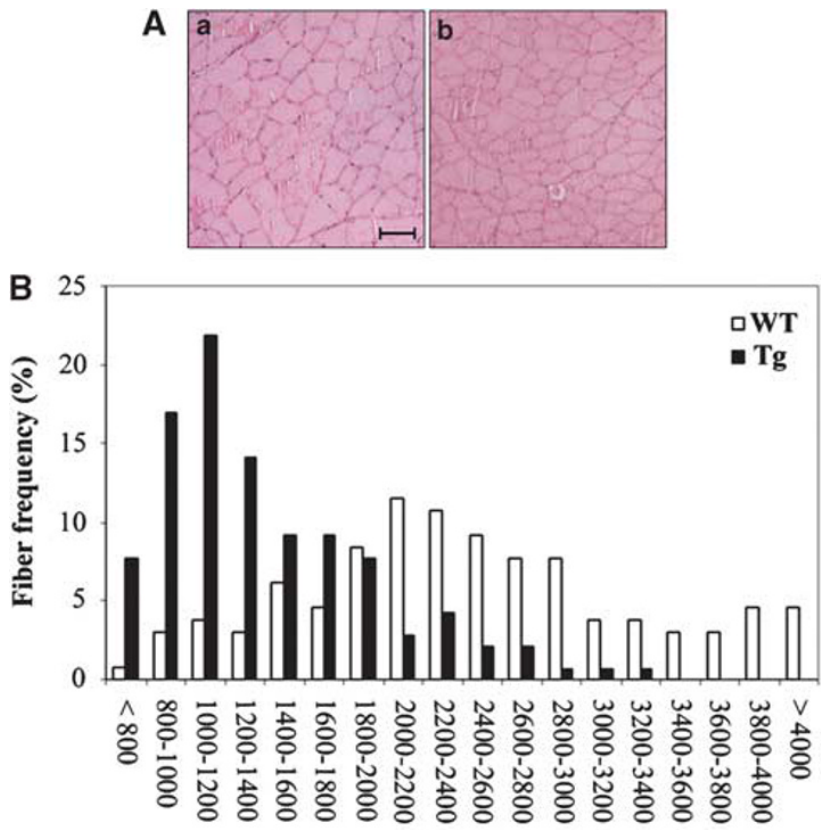

Fiber cross-sectional area $\left(\mu \mathrm{m}^{2}\right)$

Figure 2 Morphological analysis of skeletal muscle tissue sections of quadriceps muscle from Tg and WT adult mice. (A) Hemalum/eosin staining in WT (a) versus Tg (b). Bar: $50 \mu \mathrm{m}$. (B) Cross-sectional area analysis illustrating the relative abundance of different area values in $\mathrm{Tg}(N=4)$ versus WT $(N=4)$ quadriceps. Values are means \pm S.D. $P<0.05$ associated with altered enzymatic activity of the mitochondrial electron transport chain, ${ }^{17}$ and western blot analysis of quadriceps total lysates from $\mathrm{Tg}$ mice revealed a strong increase in total cytochrome $c$ content when compared with WT mice (Figure 3D). We then investigated whether mitochondrial-related alterations might be associated with oxidative damage and we found that protein carbonyl content significantly increased in adult $\mathrm{Tg}$ mice skeletal muscle when compared with the WT (1.16 \pm 0.52 versus $0.53 \pm 0.19$; Figure 3E). The observed decreased fiber size and mitochondrial dysfunction prompted us to investigate a signal transduction pathway commonly involved in aged skeletal muscle. Western blot experiments on adult $\mathrm{Tg}$ and WT skeletal muscle lysates showed decreased GSK3 $\beta$ serine-9 phosphorylation correlating with reduced Akt phosphorylation in the $\mathrm{Tg}$, whereas total Akt and GSK3 $\beta$ levels were found to be comparable (Figure 4). This result was confirmed on total lysates from different skeletal muscles (Supplementary Figure 3), such as quadriceps, soleus, tibialis and triceps, indicating a common atrophy molecular signature in different skeletal muscles. Altogether, these results show that c-Flip Tg mice show skeletal muscle atrophy associated with SDH hyper-reactive fibers without inflammatory signs but with significant oxidative damage.

Apoptosis evaluation in the adult skeletal muscle. Apoptosis was then investigated in adult $\mathrm{Tg}$ and WT skeletal muscle, as it is a candidate mechanism for satellite cell depletion in aged animals. ${ }^{18}$ In western blot experiments on cytosolic adult muscle extracts, cytochrome $c$ release was found to be increased in Tg mice when compared with the WT, thus suggesting higher apoptosis level in Tg muscles (Figure 5A). Immunohistochemistry (Figure 5B) of muscle sections showed active caspase-3-positive cells in the $\mathrm{Tg}$ but not in the WT mouse $(40 \pm 5$ positive cells per section versus $0 \pm 1$ ), clearly located between the membrane fiber and the adjacent basal lamina, with the latter recognized by antilaminin immunolabeling, indicating that apoptosis occurs in $\mathrm{Tg}$ and affects mainly satellite cells.

Analysis of regeneration after freeze injury of adult skeletal muscle. The increase in apoptosis shown above suggested that satellite cell depletions may occur in the adult $\mathrm{Tg}$ mice. To address this issue, acute injury studies on Tg versus WT were performed. The tibialis anterior (TA) muscle was damaged by freeze injury and muscles were dissected at different times after injury. As shown in Figure $6 \mathrm{~A}$, although in WT mice new myotube formation in TA muscle was observed at 7 days (Figure 6A,a), Tg mice showed delayed regeneration with fewer regenerating fibers (Figure $6 A, c$ ). The regenerative potential defect of satellite cells was also investigated at the biochemical level; proliferating cell nuclear antigen (PCNA) and embryonic myosin heavy chain (eMHC, a known regeneration marker in the adult) were analyzed by western blot in muscle samples after injury. As shown in Figure 6B, at 7 days after injury PCNA levels were lower in Tg mice, confirming a more active proliferation in the WT as further confirmed by PCNA immunohistochemical staining (Supplementary Figure 4). Furthermore, at the same time after injury, eMHC is 

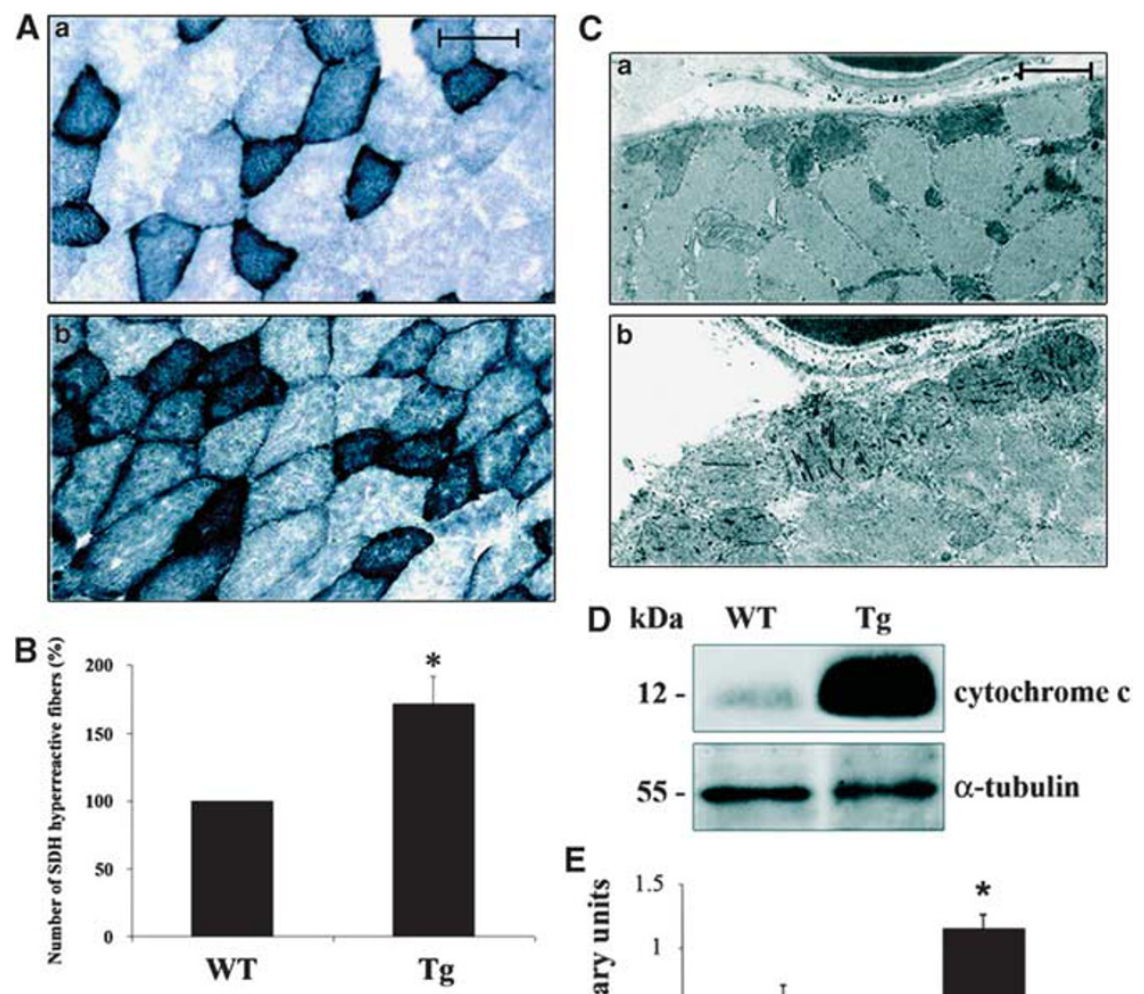

E

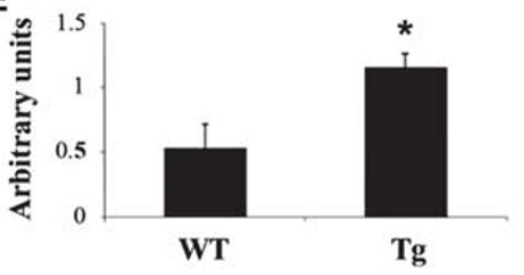

Figure 3 (A) Histochemical analysis of skeletal muscle from adult Tg and WT. Succinate dehydrogenase (SDH) activity in Tg quadriceps sections (b) when compared with the WT (a). Bar: $50 \mu \mathrm{m}$. (B) The percentage of SDH hyper-reactive fibers in the Tg (average \pm S.D.) versus WT is shown in the histogram. $N=4 .{ }^{*} P<0.05$. (C) Ultrastructure of quadriceps from adult WT (a) and Tg (b). Bar:1 1 m. (D) Anti-cytochrome $c$ western blot analysis in adult quadriceps total lysates from Tg versus WT. Data shown are representative of four independent experiments. (E) Protein carbonyl content in quadriceps extracts of Tg versus WT. Values, expressed as arbitrary units, are reported as average \pm S.E. $N=5 .{ }^{*} P<0.05$

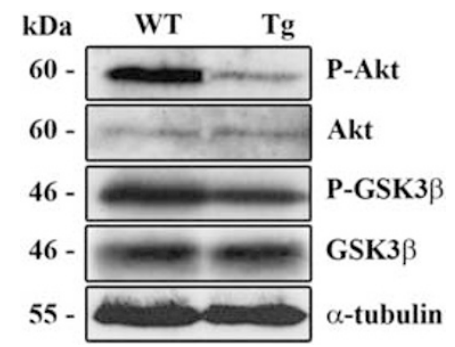

Figure 4 P-Akt, Akt, P-GSK-3 $\beta$, GSK-3 $\beta$ and $\alpha$-tubulin western blot analysis of quadriceps muscle from adult $\mathrm{Tg}$ and WT. Data shown are representative of three independent experiments

expressed at lower level in Tg versus WT mice, showing delayed regeneration in the $\mathrm{Tg}$. Taken together, these findings suggest that in the $\mathrm{Tg}$ mouse muscle regenerative activity is compromised because of impairment of satellite cells. This conclusion was further supported by immunohistochemical staining for the satellite cell marker Pax-7, which showed fewer positive cells in the Tg (Figure 6C, $\mathrm{c}$ and d) versus WT (Figure $6 \mathrm{C}$, a and b) at 4 days after injury. Quantification of Pax-7-positive cells in regenerating WT and
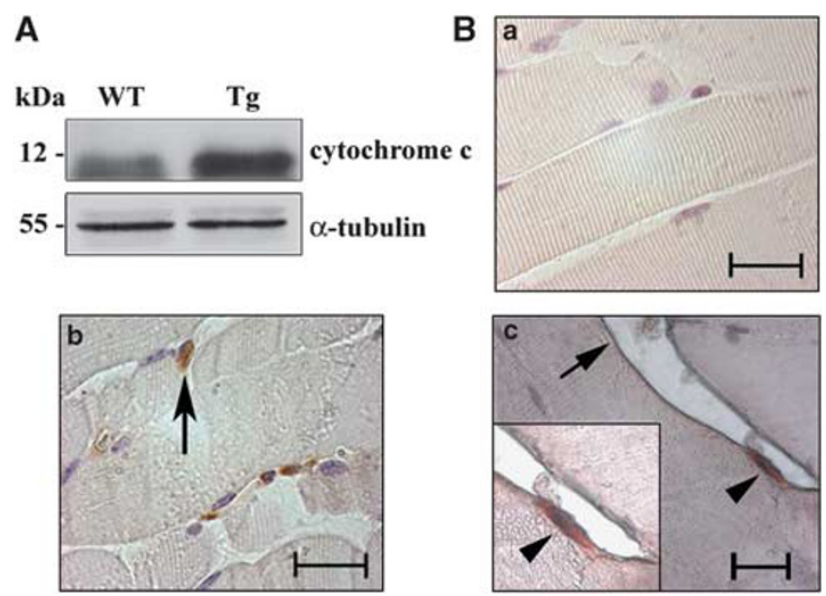

Figure 5 Apoptosis evaluation. (A; Western blot) Cytochrome $c$ content in cytosolic extracts of adult quadriceps from $\mathrm{Tg}$ and WT. Data shown are representative of three independent experiments. (B) Active caspase-3 immunohistochemistry in WT adult quadriceps (a) when compared with the Tg (b). Doubleimmunohistochemistry anti-active caspase-3 and anti-laminin on $\mathrm{Tg}$ adult quadriceps sections; the arrow indicates laminin whereas the arrowheads indicate caspase-3-positive cell underneath the laminin immunostained basal lamina (c). Bars: $20 \mu \mathrm{m}$. Sections were counterstained with hemalum 
A
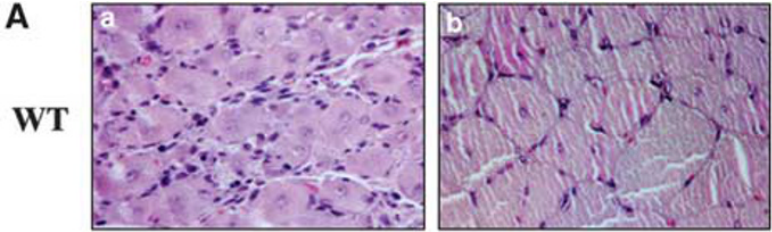

Tg

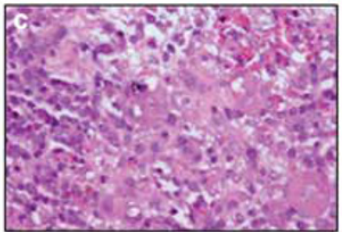

B

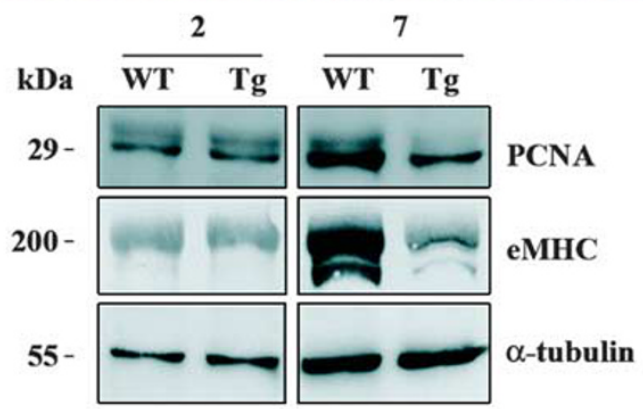

C
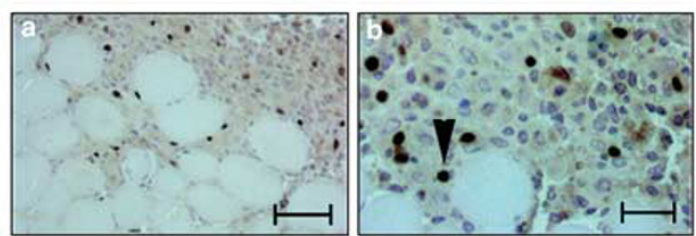

$\operatorname{Tg}$
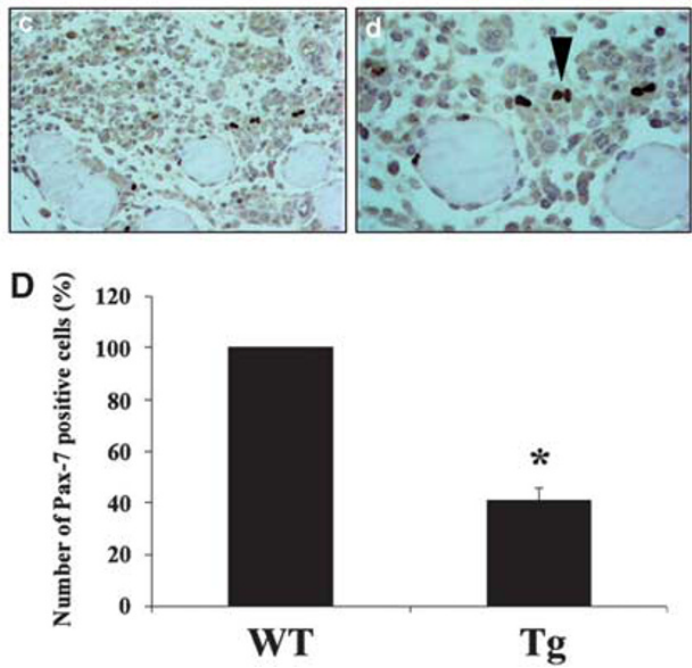

Figure 6 Regeneration experiments. (A) Morphological analysis. Hemalum/ eosin stained sections of TA from WT 7 (a) and 14 days (b) after freeze injury and $\operatorname{Tg} 7$ (c) and 14 days (d) after freeze injury. Bar: $50 \mu \mathrm{m}$. (B) Biochemical analysis. PCNA and eMHC western blot of TA from Tg and WT mice at 2 and 7 days after freeze injury. $\alpha$-Tubulin is shown for loading control. Data shown are representative of three independent experiments. (C) Pax-7 staining in WT $(\mathrm{a}, \mathrm{b})$ and $\mathrm{Tg}(\mathrm{c}, \mathrm{d})$ muscles at 4 days after injury. Arrowheads indicate Pax-7-positive nuclei. Bars: $50 \mu \mathrm{m}(\mathrm{a}, \mathrm{c})$ and $20 \mu \mathrm{m}$ (b, d). (D) The percentage of Pax-7-positive cells in the Tg (average \pm S.D.) versus WT is shown in the histogram. $N=4 .{ }^{*} P<0.05$
Tg mice is shown in Figure 6D. In addition, at 14 days after injury, normal muscle architecture seemed to be restored in WT mice (Figure 6A, b), although large areas containing little signs of newly formed muscle fibers were found in the $\mathrm{Tg}$ (Figure 6A, d). Altogether, these data indicate that forced C-Flip expression leads to impaired regenerative potential that correlates with higher apoptosis level of satellite cells(Figure 5B) and reduced Pax-7 staining (Figures $6 \mathrm{C}$ and $\mathrm{D}$ ).

Satellite cell proliferation and myogenic differentiation in infant and young mice. The data reported above suggested depletion of satellite cells in the adult c-Flip transgenic mouse. This depletion in the adult may derive from an increased proliferation of satellite cells in the infant phase, leading to the exhaustion of proliferative potential. This hypothesis was supported by the occurrence in infant $\mathrm{Tg}$ muscle of $\mathrm{C}$-Flip $\mathrm{L}$ cleavage products (Figure 1B), described as proliferation inducers. ${ }^{13}$ We thus studied the effects of c-Flip $\mathrm{L}_{\mathrm{L}}$ overexpression during the growth phase of skeletal muscle, when the supply of new myonuclei to the growing fibers is accomplished through intense satellite cell proliferation. Muscle sections of infant $\mathrm{Tg}$ mice (10 days after birth (d.a.b.)) showed a twofold increase in Pax-7-positive nuclei in Tg when compared with WT mice (Figure 7A), suggesting that c-Flip overexpression may further stimulate proliferation of satellite cells in skeletal muscle after birth. Western blot analyses of skeletal muscle infant lysates showed significantly higher levels of phosphorylated ERK (P-ERK) in Tg when compared with WT (Figure 7B), thus suggesting the involvement of ERK signaling pathways in c-Flip-driven increased proliferation, according to previous studies. ${ }^{19}$ We further observed the c-Flip-dependent proliferative activity in infant muscle extracts by PCNA analysis; PCNA was found to be expressed at higher levels in Tg versus WT mice (Figure 7B). Furthermore, in infant muscle extracts Pax-7 upregulation was confirmed, suggesting that satellite cells are increased by a proliferative mechanism (Figure 7B). Altogether, these results show that skeletal muscle overexpressing $\mathrm{C}-\mathrm{Flip}_{\mathrm{L}}$ shows hyperproliferation signs at both the morphological and biochemical levels. A concomitant increase in active caspase- 3 was observed in western blots of lysates of $\mathrm{Tg}$ when compared with WT (Figure 7B). We therefore ruled out that Pax-7 increase in $\mathrm{Tg}$ infant samples was because of c-Flipdependent apoptosis inhibition. In $\mathrm{C} 2 \mathrm{C} 12$ myoblast cell line, NF- $\kappa \mathrm{B}$ is a crucial factor for cell cycle progression into $S$ phase,$^{20}$ and cleaved $\mathrm{C}-\mathrm{Flip}_{\mathrm{L}}$ products have been shown to activate NF- $\kappa \mathrm{B} .{ }^{21}$ Therefore, to further characterize the proliferative effects of $c-F$ lip $L$ expression at the molecular level, we performed anti-phosphorylated p65 (P-p65) western blot experiments on muscle lysates at different ages (neonatal: 5 d.a.b.; infant: 10 d.a.b.; and young: 20 d.a.b.). This marker of NF- $\kappa \mathrm{B}$ activation ${ }^{22}$ was found to be strongly induced in $\mathrm{Tg}$ samples at the different time points examined (Figure 7C). To investigate whether c-Flip overexpression may cause a delay in differentiation, we monitored eMHC expression on skeletal muscle lysates from different ages. Interestingly, we found that eMHC expression in the infant was higher in $\mathrm{Tg}$ than in WT skeletal muscle lysates, and was absent in the 

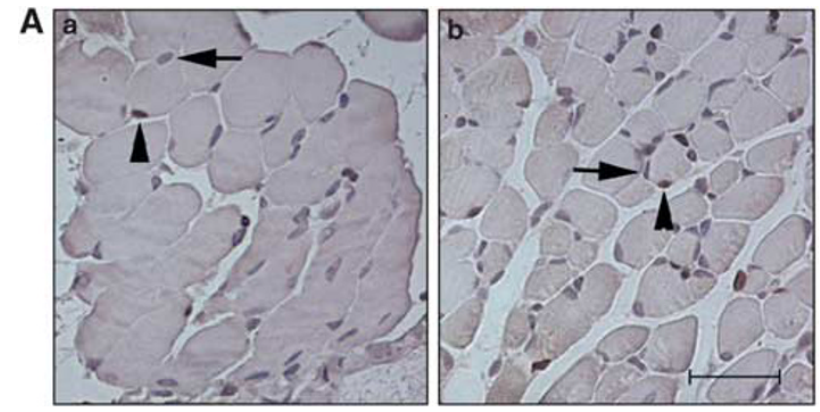

B KDa WT Tg

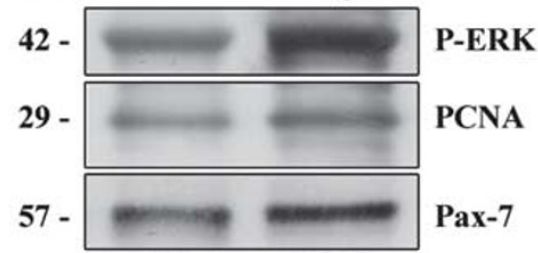

17 -

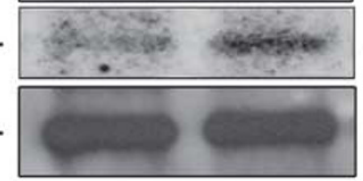

Active Caspase-3

36 neonatal

C
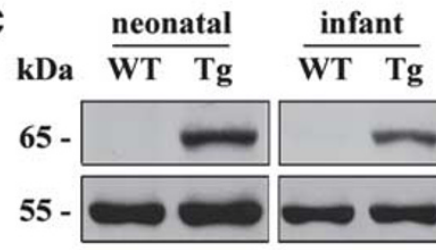

GAPDH

D
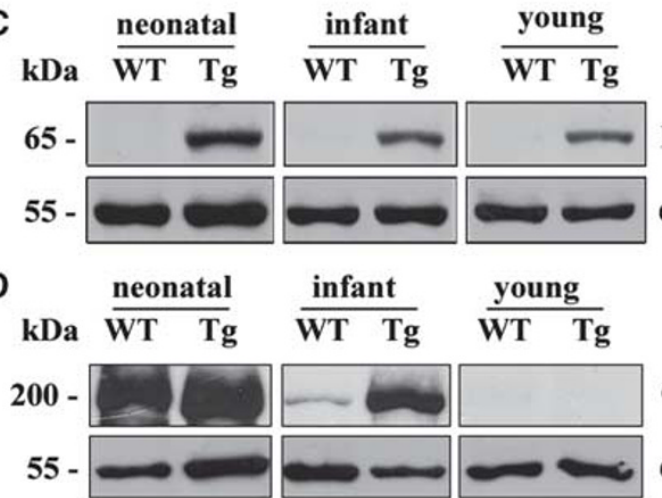

P-p65

$\alpha$-tubulin
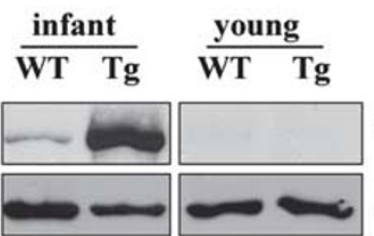

eMHC

$\alpha$-tubulin

Figure 7 (A) Anti-Pax-7 immunohistochemistry on Tg infant quadriceps (b) versus WT (a). The arrows indicate negative blue counterstained nuclei, whereas the arrowheads indicate Pax-7-positive brown nuclei. Sections were counterstained with hemalum. Bar: $60 \mu \mathrm{m}$. (B) P-ERK, PCNA, Pax-7, active caspase-3 and GAPDH western blot analysis of quadriceps from Tg and WT infant. Data shown are representative of three independent experiments. (C) P-p65 Western blot analysis of quadriceps from Tg and WT mice at different ages. $\alpha$-Tubulin staining is shown for loading control. Data shown are representative of three independent experiments. (D) eMHC and $\alpha$-tubulin western blot analyses of quadriceps from Tg and WT mice at different ages. Data shown are representative of three independent experiments

young in both WT and $\mathrm{Tg}$ (Figure 7D), indicating that myofiber maturation is delayed in vivo in Tg mice. Altogether, these data indicate that c-Flip overexpression causes myogenesis alteration through increased proliferation in the postnatal phase affecting ERK and NF- $\kappa \mathrm{B}$ signaling.

Myotube growth analysis. The increased proliferation rate and $\mathrm{eMHC}$ expression found in the infant muscles suggested that muscle growth may be perturbed. Therefore, both CSA and fiber numbers of quadriceps from infant Tg mice were measured. Muscle fibers from Tg mice showed different size
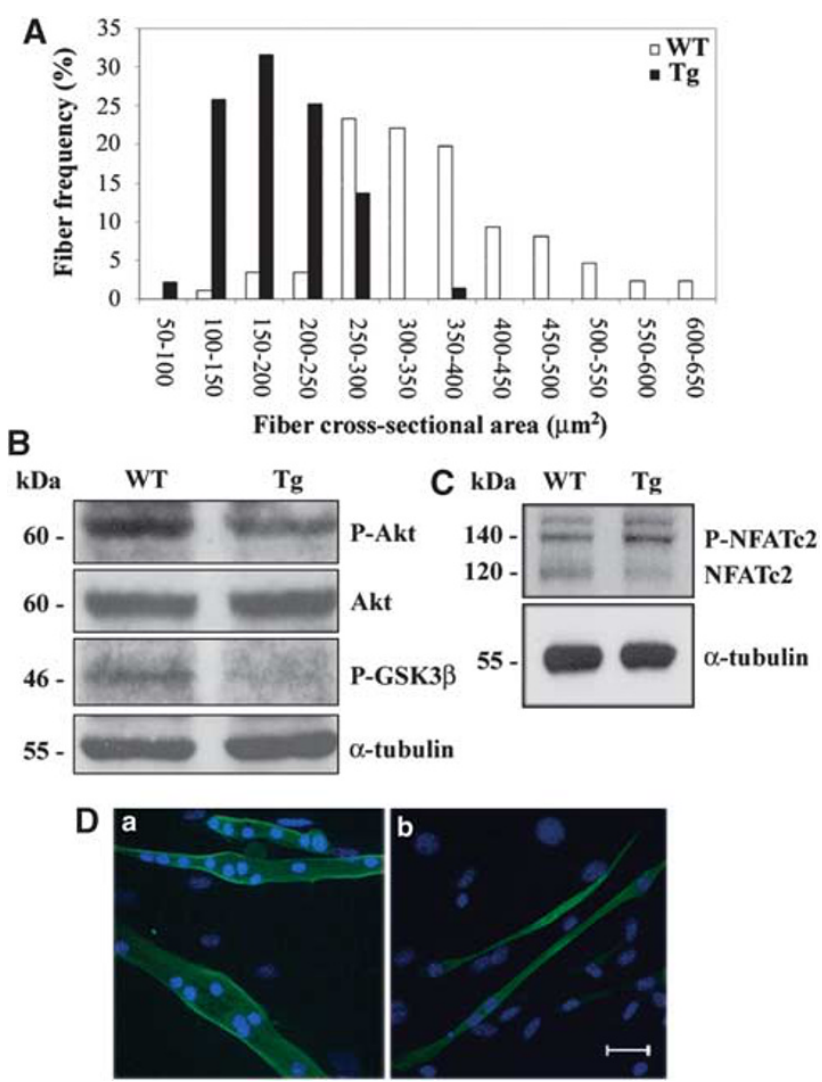

Figure 8 (A) The cross-sectional area analysis illustrating the distribution of different area values in infant $\operatorname{Tg}(N=4)$ versus WT $(N=4)$ quadriceps. Values are means \pm S.D. $P<0.05$. (B) P-Akt, Akt, P-GSK-3 $\beta$ and $\alpha$-tubulin western blot analysis of quadriceps from infant $\mathrm{Tg}$ and WT. Data shown are representative of three independent experiments. (C) P-NFATc2, NFATc2 and $\alpha$-tubulin western blot analysis of skeletal muscle from infant $\mathrm{Tg}$ and WT. Data shown are representative of three independent experiments. (D) Anti-myosin heavy chain (green) immunofluorescence on myotubes from WT (a) and $\mathrm{Tg}$ (b). Cell nuclei were evidenced with Hoechst. Bar: $50 \mu \mathrm{m}$

distribution with a shift toward smaller areas corresponding to $42.7 \%$ reduction in fiber size when compared with WT (Figure 8A), whereas no significant differences in the amount of fibers were found. As fiber growth is strongly dependent on cell fusion, we hypothesized an impairment of such process. In the infant Tg mice, similar to the adult, decreased GSK3 $\beta$ serine-9 phosphorylation correlating with reduced Akt phosphorylation was found, in the presence of comparable total Akt levels (Figure 8B). We then focused on the GSK-3 $\beta$ target NFATc2, a key transcription factor regulating myoblast fusion and fiber growth. It has been previously shown that GSK-3 $\beta$ may negatively regulate NFAT transcriptional activity. ${ }^{23}$ To better investigate the mechanism underlying the reduction of CSA in our mouse model, NFATc2 expression was investigated by western blot experiments on infant $\mathrm{Tg}$ lysates to analyze both the phosphorylated and the de-phosphorylated forms, that is, inactive and active forms. In whole tissue lysates from infant Tg skeletal muscle, the phosphorylated (inactive) form was increased whereas the dephosphorylated (active) form was decreased when compared with WT (Figure $8 \mathrm{C}$ ). This result correlates with GSK-3 $\beta$ data and leads us to hypothesize that c-Flip 
overexpression may also interfere with myoblast fusion. To address this point better, we performed satellite cell primary cultures, and cell morphology was examined daily after differentiation induction. At 1 day after medium shift, cultures from $\mathrm{Tg}$ mice formed higher numbers of thin myotubes with 2-5 nuclei when compared with cultures from WT (respectively, 80 versus $50 \%$ myotubes), as shown in Figure 8D, indicating that c-Flip overexpression, besides promoting satellite cell proliferation, negatively affects cell fusion through AKT/GSK3 $\beta /$ NFATc2pathway.

\section{Discussion}

Age-related wasting and decreased potential of muscle regeneration have been associated with impairment of satellite cells, as components of the microenvironment and intrinsic features of satellite cells may vary with age. ${ }^{1}$ This study shows that c-Flip overexpression in developing skeletal muscle induces dysregulation of muscle physiology by stimulating proliferation of myogenic cells in perinatal muscle that leads to consequent apoptosis and impaired fusion, resulting in fiber hypotrophy and premature exhaustion of the myogenic cell pool. This is the first study to investigate the effects of c-Flip forced expression in undifferentiated muscle cells in vivo; previous works have shown that c-Flip is expressed in myofibers, ${ }^{24,25}$ and that its expression is higher in aged muscles. ${ }^{26}$ The present work, carried out on a transgenic mouse overexpressing C-Flip ${ }_{L}{ }^{14}$ shows the expression of $c-F l i p_{L}$ products in satellite cells. These products of post-translational c-Flip processing are known to promote ERK and NF- $\mathrm{KB}$ proliferative signaling pathways. ${ }^{19,21}$ In $\mathrm{C} 2 \mathrm{C} 12$ myoblast cell line, NF- $\kappa$ B stimulates cell cycle progression into $\mathrm{S}$ phase ${ }^{20}$ and in different cellular systems ERK activation is crucial to stimulate proliferation. ${ }^{27}$ In this study, myogenic cell hyperproliferation was observed during the early postnatal phase of c-Flip $\mathrm{Tg}$ mice, with increased Pax-7 and higher levels of PCNA, P-ERK and P-p65. This shows that c-Flip overexpression stimulates proliferation of skeletal muscle precursors by activating ERK and NF- $\kappa \mathrm{B}$ pathways. The concomitant infant higher apoptosis level, as shown by increased active caspase-3 levels, may at least in part explain the absence of skeletal muscle hyperplasia. Furthermore, eMHC expression is more pronounced in $\mathrm{Tg}$ mice, showing that increased proliferation is associated with persistence of more immature myofibers in Tg infant mice than in WT, as shown in other transgenic models. ${ }^{28}$ Remarkably, the size of the fibers in the infant $\mathrm{Tg}$ is reduced, suggesting alteration of skeletal muscle growth. This issue was addressed focusing on the signaling pathway that is dependent on Akt phosphorylation, usually involved in hypertrophic growth, which showed reduction of both P-Akt and P-GSK in the infant Tg muscles. In addition, the GSK-3 $\beta$ target NFATc2, a key transcription factor regulating myoblast fusion and fiber growth, was investigated. It is known that muscle cells defective in NFATc2 form small thin myotubes with few nuclei because of the inhibition of myoblast cell fusion with nascent myotubes. ${ }^{29}$ Previous studies performed on $\mathrm{C}-\mathrm{Flip}_{\mathrm{L}}$ overexpressing $\mathrm{T}$ cells showed reduced active NFATc2 levels. ${ }^{30}$ We show that c-Flip overexpression, through GSK-3 $\beta$, stimulates the phosphorylation of NFATc2, and hence the inactivation, thus reducing cell fusion. We conclude that the CSA reduction observed in infant $\mathrm{Tg}$ mice may be because of an impairment in fiber size growth. The analysis of adult $\mathrm{Tg}$ mice revealed that skeletal muscles are characterized by marked reduction of fiber CSA and by mitochondrial alterations, namely, increase in SDH and total cytochrome $c$ levels. Mitochondrial abnormalities are observed in muscles undergoing sarcopenia, defined as loss of muscle mass with age. Indeed, aging associates with mitochondrial DNA mutations and with electron transport abnormalities, leading to abnormal mitochondria proliferation beneath the sarcolemma. ${ }^{31}$ We therefore hypothesize that the increased cytochrome $c$ level in total skeletal muscle lysates of our $\mathrm{Tg}$ model is related to increased mitochondrial proliferation in line with ultrastructural indications. Protein carbonylation is a prominent marker of oxidative stress in aged skeletal muscle and was found to be increased in the $\mathrm{Tg}$ muscle lysates, thus indicating a correlation between mitochondrial modifications and oxidative stress. Adult $\mathrm{Tg}$ skeletal muscles also show downregulation of both P-Akt and $\mathrm{P}-\mathrm{GSK} 3 \beta$. Akt is involved in the decreased protein synthesis associated with muscle atrophy, and GSK3 $\beta$ activation (through reduction in the serine 9 inhibitory phosphorylation) is known to be involved in muscle atrophy. ${ }^{32}$ The involvement of GSK3 $\beta$ activation in mitochondrial apoptosis, ${ }^{33}$ as well as the role apoptosis has in skeletal muscle atrophy, may correlate, at least in part, with the increased cytochrome $c$ release in the cytosol and caspase- 3 activation observed in the $\mathrm{Tg}$. It is known that atrophy may occur in the absence of myonuclear loss but it is accompanied by increased apoptosis of stromal and satellite cells. ${ }^{34}$ In this study we found cells recognized by active caspase- 3 antibodies located external to the plasmalemma and underneath the basal lamina of $\mathrm{Tg}$ mouse but no immunostaining inside the muscle fibers, indicating that in our model the apoptotic program primarily affects the satellite cells. Altogether, the observed muscle alterations, that is, reduced fiber areas, histochemical, biochemical and ultrastructural signs of mitochondrial alteration, elevation of oxidative damage and activation of biochemical atrophic signaling, represent typical modifications responsible for the decline in muscle quality within aged muscles. We conclude that the increased proliferation of satellite cells during the early postnatal phase may lead to the observed impaired regeneration in the adult, through satellite cell proliferative potential reduction, as well as to aged phenotype. Proliferative senescence has been already implicated in the loss of regenerative capacity in satellite cells from Duchenne dystrophic skeletal muscles and may participate in the atrophy of myotonic dystrophy type $1 .{ }^{35}$ Our data show that c-Flip-induced atrophic phenotype correlates with both myogenic precursors hyperproliferation (leading to premature senescence) and with the alteration of Akt/GSK3 $\beta$ phosphorylation status. A regulatory role of c-Flip in cell proliferation in mammalian cell lines has been recently reported. It has been shown that c-Flip rapidly translocates to the nucleus in response to proliferative stimuli, and evidence is given that c-Flip-dependent proliferative effect may occur through transcriptional regulation. ${ }^{36}$ On the other hand, Fas signaling mediates hypertrophic signaling, regardless of Fas ligand stimulation, through GSK3 $\beta$ phosphorylation. ${ }^{37}$ 
This study on c-Flip, a well-known Fas signaling modulator, supports this view. In conclusion, we believe that this mouse model, overexpressing c-Flip in skeletal muscle under trStra8 promoter, may help to clarify the molecular bases of muscle physiology, elucidating a potential novel role of c-Flip in the control of satellite cell proliferative potential and muscle mass loss in disease or aging.

\section{Materials and Methods \\ Animals. c-Flip transgenic mice generation was previously described. ${ }^{14,38,39}$ The 400 bp Stra8 promoter ${ }^{40}$ was kindly provided by F Cuzin (Inserm, Nice, France) and cloned in $\mathrm{pBS}$ SK vector. Mouse C-Flip $p_{L}$ sequence was kindly provided by M Peter (The Ben May Institute for Cancer Research, University of Chicago, Chicago, IL USA) and was cloned into the pBS SK vector. IRES-EGFP sequence was excised from the pIRES2-EGFP vector (Stratagene, La Jolla, CA, USA) and cloned downstream of $\mathrm{c}$-Flip $\mathrm{L}$. The complete expression cassette from the pBS SK vector was excised and injected into FVB $\times$ FVB single-cell-stage embryos. Experiments were carried out on neonatal mice ( 5 d.a.b.), infant mice (10 d.a.b.), young mice (20 d.a.b.) and adult mice (4 months). Animal experiments were carried out in accordance with the European Communities Council Directive of 24 November 1986 (86/609/EEC). The experimental protocol was approved by the Italian Health Ministry.}

RNA isolation and RT-PCR. Mouse tissues (skeletal muscle and heart) were added directly to Trizol (Invitrogen, San Giuliano Milanese, Italy) and homogenized, and RNA isolation was performed according to the manufacturer's protocol. RNA $(2 \mu \mathrm{g})$ was used, after DNAse incubation aimed to remove genomic DNA, in a reverse transcription (RT) reaction by using SuperScript II Reverse Transcriptase (Invitrogen); then RT samples were used in a PCR reaction performed with recombinant Taq DNA polymerase (Invitrogen) in the presence of the EGFP primers.

The EGFP primers were the following: $5^{\prime}$-GTAAACGGCCACAAGTTC- $3^{\prime}$ and $5^{\prime}$-CAAGGTGAACTTCAAGATCC- $3^{\prime}$. For normalization, control mouse $\beta$-actin primers were used under parallel conditions.

Western blotting. Lysates were prepared with Cell Lysis Buffer (New England Biolabs, Beverly, MA, USA) containing PMSF (Sigma Chemical Co., St. Louis, MO, USA). The following skeletal muscles were homogenized: quadriceps, soleus, tibialis and triceps. Equal amounts of proteins were subjected to SDSpolyacrylamide gel electrophoresis. Equal loading was checked by anti- $\alpha$-tubulin or glyceraldehyde-3-phosphate dehydrogenase (GAPDH).

To evaluate cytochrome $c$ release from mitochondria, cytosolic extracts were prepared by skeletal muscle homogenization in MES buffer (Sigma Chemical Co.) followed by $10000 \times g$ centrifugation to remove mitochondria.

Protein concentration was determined using the micro-BCA method (Pierce, Tattenhall, Cheshire, UK). Secondary antibodies were horseradish peroxidase conjugated (Pierce, Rockford, IL, USA; Alexis, Lausen, Switzerland); detection was performed using standard or enhanced chemiluminescence measurement (ECL; Amersham Pharmacia Biotech, Buckinghamshire, UK).

The following antibodies were used: anti-tubulin mouse monoclonal IgG (Sigma Chemical Co.), anti-GAPDH, anti-phosphoGSK3 $\beta$ (Ser9), anti-GSK3 $\beta$, anti-phosphoAkt (Ser 473), anti-Akt, anti-phospho-p65 and anti-phosphoERK (Cell Signaling, Denvers, MA, USA), anti-Pax7 and anti-eMHC (Hybridoma Bank, lowa City, IA, USA), anti-cytochrome $c$ (R\&D, Minneapolis, MN, USA), anti-Flip Dave-II (Alexis) and anti-PCNA (Abcam, Cambridge, UK).

Cell cultures. C2C12 satellite cell-derived cell line was cultured in DMEM (Sigma) supplemented with 10\% FCS (Sigma), $2 \mathrm{mM}$ L-glutamine (Sigma Chemical Co.) and maintained in $5 \% \mathrm{CO}_{2}$ at $37^{\circ} \mathrm{C}$. To prepare satellite cells, muscle fragments were digested with collagenase dispase (Roche Diagnostics $\mathrm{GmbH}$, Mannheim, Germany) for $20 \mathrm{~min}$ at $37^{\circ} \mathrm{C}$ and then with collagenase II (Sigma Chemical Co.) for $10 \mathrm{~min}$ at $37^{\circ} \mathrm{C}$ with gentle agitation. Contamination by nonmyogenic cells was reduced by pre-plating the cell suspension onto plastic dishes in which fibroblasts tend to adhere more rapidly. Isolated cells were cultured in DMEM supplemented with $20 \%$ horse serum and $3 \%$ chicken embryo extract. Differentiation was induced shifting the medium to DMEM supplemented with $2 \%$ horse serum.
Morphologic analysis. For light microscopy analysis of skeletal muscle, the specimens $(N=4$ for both WT and $\mathrm{Tg}$ ) were frozen in liquid nitrogen or formalin fixed and paraffin embedded. Cryostat sections were analyzed by enzyme histochemistry to determine SDH activity. For SDH quantification, the hyper-reactive fibers were counted on 20 fields from both WT and Tg muscle sections. Paraffin samples were sectioned and histologically stained with Carazzi hemallum or hemalum/eosin. The fiber CSRs were measured using the Scion image software (Scion Corp., Frederick, MD, USA). In particular, these morphometric analyses were performed on sections collected from similar regions of quadriceps muscles obtained from four WT and four Tg mice. Two images were captured from each section and the software was used to determine the CSA of 200-400 myofibers per sample.

Immunohistochemical staining. Skeletal muscle samples $(N=4$ for both WT and $\mathrm{Tg}$ ) were fixed in formalin solution, dehydrated and embedded in paraffin wax before sectioning. Serial sections $(3 \mu \mathrm{m})$ were mounted on Superfrost Plus slides (Menzel, GmbH \& Co., Braunschweig, Germany). In brief, tissue sections were deparaffinized in xylene, rehydrated in graded ethanols and treated with methanol containing $0.3 \% \mathrm{H}_{2} \mathrm{O}_{2}$ to block endogenous peroxidase activity. Slides, in citrate buffer, were microwaved for $10 \mathrm{~min}$. Immunohistochemical staining was performed through Histostain-Plus (Zymed Laboratories South San Francisco, CA, USA and Invitrogen) according to the manufacturer's instructions. The following antibodies were used: anti-EGFP (Molecular Probes, Milan, Italy and Invitrogen), anti-dystrophin (Menarini Diagnostics, Firenze, Italy), anti-cleaved caspase-3 (Abcam), anti-laminin (Sigma) and anti-PCNA (Abcam). As a control for the immunohistochemistry method, the primary antibody was omitted and no staining was detected under these conditions.

Microscope (Axioskop 2 plus; Carl Zeiss Microimaging, Inc.) with $\times 10$ NA 0.30 , $\times 20$ NA $0.50, \times 40$ NA 0.75 or $\times 63$ NA 1.25 oil objective lenses (Plan-Neofluar) was used. Images were obtained at room temperature using AxioCamHRC camera (Carl Zeiss Microimaging, Inc., Milan, Italy) by Axiovision 3.1 software and images were assembled in panels using Photoshop 7.0 (Adobe, Waltham, MA, USA).

Electron microscopy. For electron microscopy, specimens ( $N=4$ for both WT and Tg) were fixed in $2.5 \%$ glutaraldehyde in $0.2 \mathrm{M}$ phosphate buffer $\mathrm{pH} 7.2$, postfixed in $1 \% \mathrm{OsO}_{4}$ and embedded in Epon. All reagents for morphological analysis were purchased from Bio-Optica (Milano, Italy). Sections were prepared from the embedded samples and examined under the electron microscope, model CM10 Philips (Eindhoven, Holland). Hewlett-Packard Digital Imaging software (Houston, TX, USA) was used to generate images.

Protein oxidation evaluation. Protein oxidation was evaluated through protein carbonyl content in skeletal muscle homogenates using Protein carbonyl Assay kit (CAYMAN Chemical, Ann Arbor, MI, USA) according to the manufacturer's instructions.

Fluorescence analysis. At $24 \mathrm{~h}$ after plating, $\mathrm{C} 2 \mathrm{C} 12$ cells were transiently transfected with $1 \mu \mathrm{g}$ tr-Stra8-EGFP vector using Lipofectamine plus (Invitrogen) according to the manufacturer's instructions. At $48 \mathrm{~h}$ after transfection, EGFP fluorescence was visualized.

For fluorescence microscopy analysis of myogenic primary cell cultures $(N=3$ from both WT and Tg quadriceps) under differentiative conditions, culture dishes were washed with PBS, fixed with $4 \%$ paraformaldehyde for $10 \mathrm{~min}$ and then permeabilized with $0.2 \%$ Triton X-100 in PBS for 5 min. After incubation with PBS containing $10 \%$ normal goat serum, cells were incubated with anti-myosin heavy chain (MF20) antibody in a 1:10 dilution for $1 \mathrm{~h}$ at room temperature. After incubation, cells were washed and incubated with goat anti-mouse FITC-conjugated secondary antibodies for $1 \mathrm{~h}$ at room temperature. Cell nuclei were evidenced with Hoechst. As a control for immunofluorescence the primary antibody was omitted and no fluorescence was detected under these conditions.

Induction of muscle regeneration. Mice were anesthetized with an intramuscular injection of a cocktail of $35 \mathrm{mg} / \mathrm{kg}$ ketamine and $5 \mathrm{mg} / \mathrm{kg}$ xylazine and a small cut was made to expose the TA muscles. The muscle was subjected to three consecutive cycles of freezing and thawing by applying a liquid nitrogen-cooled metallic rod. The TA muscles $(N=4)$ were isolated at various time points $(2,4,7$ and 14 days) after the freeze injury. The mice were killed by cervical dislocation. The TA muscles from untreated mice were used as a control. Samples were collected for histological and immunohistochemical analysis and western blotting. 
For Pax-7 quantification during regeneration, the Pax-7-positive cells were counted on 20 fields from both WT and Tg.

Statistical analysis. Values are expressed as means \pm S.D. Statistical evaluation was performed using Student's $t$-test analysis and a value of $P<0.05$ was considered statistically significant.

\section{Conflict of Interest}

The authors declare no conflict of interest.

Acknowledgements. We gratefully thank Professor S Schiaffino and Professor A Musarò for helpful discussion, Dr. P Smeriglio for support in regeneration experiments, Dr. A D'Alessio for supporting graphics preparation and Professor F Palombi and Dr. A Facchiano for critical reading of the paper. This work was supported by the Italian Ministry of Health Grant 2007 and by the MIUR-PRIN Grant 2007

1. Brack AS, Rando TA. Intrinsic changes and extrinsic influences of myogenic stem cell function during aging. Stem Cell Rev 2007; 3: 226-237.

2. Schultz E, Lipton BH. Skeletal muscle satellite cells: changes in proliferation potential as a function of age. Mech Ageing Dev 1982; 20: 377-383.

3. Lexell J. Human aging, muscle mass, and fiber-type composition. J Gerontol A Biol Sci Med Sciences 1995; 50: 11-16.

4. Dirks A, Leeuwenburgh C. Apoptosis in skeletal muscle with aging. Am J Physiol Regul Integr Comp Physiol 2002; 282: R519-R527.

5. Gupte AA, Bomhoff GL, Geiger PC. Age-related differences in skeletal muscle insulin signaling: the role of stress kinases and heat shock proteins. J Appl Physiol 2008; 105 : 839-848.

6. Thompson LV. Age-related muscle dysfunction. Exp Gerontol 2009; 44: 106-111.

7. Decary S, Ben Hamida C, Mouly V, Barbet JP, Hentati F, Butler-Browne GS. Shorter telomeres in dystrophic muscle consistent with extensive regeneration in young children. Neuromuscul Disord 2000; 10: 113-120.

8. Seal S, Hockenbery DM, Spaulding EY, Kiem HP, Abbassi N, Deeg HJ. Differentia responses of FLIPLong and FLIPShort-overexpressing human myeloid leukemia cells to TNF-alpha and TRAlL-initiated apoptotic signals. Exp Hematol 2008; 36: 1660-1672.

9. Yeh WC, Itie A, Elia AJ, Ng M, Shu HB, Wakeham A et al. Requirement for Casper (c-FLIP) in regulation of death receptor-induced apoptosis and embryonic development. Immunity 2000; 12: 633-642.

10. Giampietri C, Petrungaro S, Coluccia P, D'Alessio A, Starace D, Riccioli A et al. FLIP is expressed in mouse testis and protects germ cells from apoptosis. Cell Death Differ 2003 10: $175-184$.

11. Giampietri C, Petrungaro S, Klinger FG, Coluccia $P$, Paone A, Vivarelli E et al. c-Flip expression and function in fetal mouse gonocytes. FASEB J 2005; 19: 124

12. Boatright KM, Deis C, Denault JB, Sutherlin DP, Salvesen GS. Activation of caspases-8 and -10 by FLIP(L). Biochem J 2004; 382 (Pt 2): 651-657.

13. Yu JW, Shi Y. FLIP and the death effector domain family. Oncogene 2008; 27: 6216-6227.

14. Giampietri $\mathrm{C}$, Petrungaro S, Musumeci M, Coluccia $\mathrm{P}$, Antonangeli $\mathrm{F}$, De Cesaris $\mathrm{P}$ et al. c-Flip overexpression reduces cardiac hypertrophy in response to pressure overload. $J$ Hypertens 2008; 26: 1008-1016.

15. Pavlath GK, Horsley V. Cell fusion in skeletal muscle - central role of NFATC2 in regulating muscle cell size. Cell Cycle 2003; 2: 420-423.

16. Rifai Z, Welle S, Kamp C, Thornton CA. Ragged red fibers in normal aging and inflammatory myopathy. Ann Neurol 1995; 37: 24-29.

17. Oppenheim ML, Hargreaves IP, Pope S, Land JM, Heales SJ. Mitochondrial cytochrome $c$ release: a factor to consider in mitochondrial disease? J Inherit Metab Dis 2009; 32 269-273.

18. Jejurikar SS, Henkelman EA, Cederna PS, Marcelo CL, Urbanchek MG, Kuzon WM. Aging increases the susceptibility of skeletal muscle derived satellite cells to apoptosis. Exp Gerontol 2006; 41: 828-836.

19. Kataoka T, Budd RC, Holler N, Thome M, Martinon F, Irmler M et al. The caspase-8 inhibitor FLIP promotes activation of NF-kappaB and Erk signaling pathways. Curr Biol 2000; 10: 640-648.

20. Guttridge DC, Albanese C, Reuther JY, Pestell RG, Baldwin Jr AS. NF-kappaB controls cell growth and differentiation through transcriptional regulation of cyclin D1. Mol Cell Biol 1999; 19: $5785-5799$.

21. Golks A, Brenner D, Krammer PH, Lavrik IN. The c-FLIP-NH2 terminus (p22-FLIP) induces NF-kappaB activation. J Exp Med 2006; 203: 1295-1305.

22. Kefaloyianni $E$, Gaitanaki $C$, Beis I. ERK1/2 and p38-MAPK signalling pathways, through MSK1, are involved in NF-kappaB transactivation during oxidative stress in skeletal myoblasts. Cell Signal 2006; 18: 2238-2251.

23. van der Velden JL, Langen RC, Kelders MC, Willems J, Wouters EF, Janssen-Heininger YM et al. Myogenic differentiation during regrowth of atrophied skeletal muscle is associated with inactivation of GSK-3beta. Am J Physiol Cell Physiol 2007; 292: C1636-C1644.

24. Nagaraju K, Casciola-Rosen L, Rosen A, Thompson C, Loeffler L, Parker T et al. The inhibition of apoptosis in myositis and in normal muscle cells. J Immunol 2000; 164: 5459-5465.

25. Benayoun B, Baghdiguian S, Lajmanovich A, Bartoli M, Daniele N, Gicquel E et al. NF-kappaB-dependent expression of the antiapoptotic factor C-FLIP is regulated by calpain 3, the protein involved in limb-girdle muscular dystrophy type 2A. FASEB J 2008; 22: $1521-1529$.

26. Pistilli EE, Jackson JR, Alway SE. Death receptor-associated pro-apoptotic signaling in aged skeletal muscle. Apoptosis 2006; 11: 2115-2126.

27. Ramos JW. The regulation of extracellular signal-regulated kinase (ERK) in mammalian cells. Int J Biochem Cell Biol 2008; 40: 2707-2719.

28. Perdiguero E, Ruiz-Bonilla V, Gresh L, Hui L, Ballestar E, Sousa-Victor P et al. Genetic analysis of p38 MAP kinases in myogenesis: fundamental role of p38alpha in abrogating myoblast proliferation. EMBOJ 2007; 26: 1245-1256.

29. Horsley V, Friday BB, Matteson S, Kegley KM, Gephart J, Pavlath GK. Regulation of the growth of multinucleated muscle cells by an NFATC2-dependent pathway. J Cell Biol 2001; 153: 329-338

30. Wu W, Misra RS, Russell JQ, Flavell RA, Rincon M, Budd RC. Proteolytic regulation of nuclear factor of activated T (NFAT) c2 cells and NFAT activity by caspase-3. J Biol Chem 2006; 281: 10682-10690.

31. Bua EA, McKiernan SH, Wanagat J, McKenzie D, Aiken JM. Mitochondrial abnormalities are more frequent in muscles undergoing sarcopenia. J Appl Physiol 2002; 92: 2617-2624.

32. Leger B, Cartoni R, Praz M, Lamon S, Deriaz O, Crettenand A et al. Akt signalling through GSK-3beta, mTOR and Foxo1 is involved in human skeletal muscle hypertrophy and atrophy. J Physiol 2006; 576 (Pt 3): 923-933.

33. Lin CF, Chen CL, Chiang CW, Jan MS, Huang WC, Lin YS. GSK-3beta acts downstream of PP2A and the PI 3-kinase-Akt pathway, and upstream of caspase-2 in ceramide-induced mitochondrial apoptosis. J Cell Sci 2007; 120 (Pt 16): 2935-2943

34. Bruusgaard JC, Gundersen K. In vivo time-lapse microscopy reveals no loss of murine myonuclei during weeks of muscle atrophy. J Clin Invest 2008; 118: 1450-1457.

35. Bigot A, Klein AF, Gasnier E, Jacquemin V, Ravassard P, Butler-Browne G et al. Large CTG repeats trigger p16-dependent premature senescence in myotonic dystrophy type 1 muscle precursor cells. Am J Pathol 2009; 174: 1435-1442.

36. Zhang J, Chen Y, Huang Q, Cheng W, Kang Y, Shu L et al. Nuclear localization of c-FLIP-L and its regulation of AP-1 activity. Int J Biochem Cell Biol 2009; 41: 1678-1684.

37. Badorff C, Ruetten H, Mueller S, Stahmer M, Gehring D, Jung F et al. Fas receptor signaling inhibits glycogen synthase kinase 3 beta and induces cardiac hypertrophy following pressure overload. J Clin Invest 2002; 109: 373-381.

38. Antonangeli F, Giampietri C, Petrungaro S, Filippini A, Ziparo E. Expression profile of a 400-bp Stra8 promoter region during spermatogenesis. Microsc Res Tech 2009; 72: 816-822.

39. Antonangeli F, Petrungaro S, Coluccia P, Filippini A, Ziparo E, Giampietri C. Testis atrophy and reduced sperm motility in transgenic mice overexpressing c-FLIP(L). Fertil Steril 2010; 93: $1407-1414$

40. Giuili G, Tomljenovic A, Labrecque N, Oulad-Abdelghani M, Rassoulzadegan M, Cuzin F. Murine spermatogonial stem cells: targeted transgene expression and purification in an active state. EMBO Rep 2002; 3: 753-759.

Cell Death and Disease is an open-access journal published by Nature Publishing Group. This article is licensed under a Creative Commons Attribution-Noncommercial-No Derivative Works 3.0 License. To view a copy of this license, visit http:// creativecommons.org/licenses/by-nc-nd/3.0/

\section{Supplementary Information accompanies the paper on Cell Death and Disease website (http://www.nature.com/cddis)}

